P. JÄRVI

KODAI MATH. J.

$13(1590), 84-87$

\title{
ON THE BOUNDARY BEHAVIOR OF HOLOMORPHIC MAPPINGS OF PLANE DOMAINS INTO RIEMANN SURFACES
}

\author{
By PENTTI JäRVI
}

1. Since the pioneering work of Ohtsuka ([7], [8]), several papers have dealt with Picard-type theorems for holomorphic mappings of plane domains into Riemann surfaces. See, e.g., [2], [4], [5], [6], [9], [10], [12] and [13]. In this note we shall consider the behavior around null-sets of class $N_{B}$ and $N_{D}$ (in the familiar notation of Ahlfors-Beurling [1]) of holomorphic mappings into certain Riemann surfaces. Our result concerning the class $N_{B}$ (Theorem 1 ) can be regarded as a generalization of a recent result of Shiga [12, Theorem 2].

2. We begin with some terminology. Let $W$ be a Riemann surface and $E \subset W$ a compact totally disconnected set. We say that $E$ is of class $N_{B}$ (resp. $\left.N_{D}\right)$ in $W$ if for each $p \in E$ there is a parametric disc $(V, \varphi)$ in $W$ such that $p \in V, E \cap \partial V=\varnothing$ and $\varphi(E \cap V)$ is of class $N_{B}$ (resp. $N_{D}$ ). Let $W^{*}$ stand for the Stoïlow compactification of $W$, and let $p \in \beta=W^{*} \backslash W$. We say that $p$ is $A B$ removable (resp. $A D$-removable) if there is a planar end $V \subset W$ with $p \in \beta_{V}$, the relative ideal boundary of $V$, and a conformal map $\varphi$ of $\bar{V}$ into the closed unit disc $\bar{U} \subset C$ such that $\varphi(\partial V)=\partial \bar{U}$ and $\bar{U} \backslash \varphi(\bar{V})$ is of class $N_{B}$ (resp. $N_{D}$ ). Obviously, $p \in \beta$ is $A B$-removable if and only if there is a Riemann surface $W^{\prime} \supset W$ such that $p \in W^{\prime} \backslash W$ and $W^{\prime} \backslash W$ is of class $N_{B}$ in $W^{\prime}$. As usual, $\theta_{A B}$ denotes the class of Riemann surfaces which do not carry nonconstant bounded holomorphic functions, while $\mathcal{O}_{M D^{*}}$ stands for the class of Riemann surfaces without nonconstant meromorphic functions with a finite spherical Dirichlet integral.

THEOREM 1. Let $D$ be a plane domain and let $E \subset D$ be a compact set of class $N_{B}$. Let $W$ be a Riemann surface which does not belong to $\mathcal{O}_{A B}$, and let $f: D \backslash E \rightarrow W$ be a holomorphic mapping. Then there exists a Riemann surface $W^{\prime} \supset W$ such that

(a) $W^{\prime} \backslash W$ is of class $N_{B}$ in $W^{\prime}$ and

(b) $f$ extends to a holomorphic mapping $f^{*}: D \rightarrow W^{\prime}$.

Proof. We may assume that $f$ is nonconstant. Let $g$ be a nonconstant bounded holomorphic function in $W$. Since $E \in N_{B}, g \circ f$ admits a holomorphic

Received June 26, 1989 
extension to $D$. Fix $z \in E$. Then $C l(g \circ f ; z)$, the cluster set of $g \circ f$ attached to $z$, is a singleton. Since $g$ is nonconstant, $\operatorname{Cl}(f ; z)$ must be totally disconnected. Hence, if taken with respect to the Stoïlow compactification $W^{*}, C l(f ; z)$ is a singleton. In other words, $f$ extends to a continuous mapping $f^{*}: D \rightarrow W^{*}$.

Obviously, $f^{*}$ is holomorphic in $D \backslash E^{\prime}$, where $E^{\prime}$ stands for $\left(f^{*}\right)^{-1}(\beta)$. We claim that $W^{\prime}=W \cup f^{*}\left(E^{\prime}\right)$ can be given a conformal structure, compatible with that of $W$. Fix $p_{0} \in f^{*}\left(E^{\prime}\right)$ and pick out $z_{0} \in E^{\prime}$ such that $p_{0}=f^{*}\left(z_{0}\right)$. Then choose a Jordan domain $U$ with $z_{0} \in U$ and $E^{\prime} \cap \partial U=\varnothing$, and let $V$ be an end of $W$ such that $f^{*}(\partial U) \cap \bar{V}=\varnothing$ and $p_{0} \in \beta_{V}$. Let $\left(\bar{V}_{n}\right)_{n \in N}$ be a relative exhaustion of $\bar{V}$. We may assume that $\partial V_{n}$ consists of a finite number of Jordan curves and there is no branch point of $f^{*}$ in $\left(f^{*}\right)^{-1}\left(\partial V_{n}\right) \cap U, n \in N$. Observe that the valence function of $f^{*} \mid U \backslash E^{\prime}$ is finite and constant, say $m$, in $V$. Hence $f^{*}$ defines a proper mapping of every component of $\left(f^{*}\right)^{-1}\left(V_{n}\right) \cap U$ onto $V_{n}$ for each $n \in N$. Furthermore, it is easy to see that $\left(f^{*}\right)^{-1}\left(V_{n}\right) \cap U$ is connected for large $n$. Indeed, let $p \in V$ and let $K \subset U \backslash E^{\prime}$ be a compact connected set which contains the preimages of $p$ in $U$. Clearly, $\left(f^{*}\right)^{-1}\left(V_{n}\right) \cap U$ is then connected provided $f^{*}(K) \subset V_{n}$.

Let $b_{n}\left(\right.$ resp. $\left.b_{n}^{\prime}\right)$ be the number of the boundary curves of $V_{n}\left(\operatorname{resp} .\left(f^{*}\right)^{-1}\left(V_{n}\right)\right.$ $\cap U)$, and let $g_{n}$ stand for the genus of $V_{n}$. By the Riemann-Hurwitz formula, we have

$$
b_{n}^{\prime}-2 \geqq m\left(2 g_{n}+b_{n}-2\right) \quad \text { for large } n .
$$

On the other hand, $b_{n}^{\prime} \leqq m b_{n}$ for such $n$. Hence $m g_{n} \leqq m-1$, whence $g_{n} \leqq$ $(m-1) / m<1$. Thus, $V_{n}$ is planar for each $n \in N$. In other words, we may realize $V$ as a plane domain bounded by a finite number of Jordan curves (corresponding to $\partial V$ ) and a closed set $F$ (corresponding to $\beta_{V}$ ). Clearly, all that remains is to show that $F$ is of class $N_{B}$. But this follows from the corresponding property of $E^{\prime}$ and the fact that a nonconstant holomorphic function is a local homeomorphism off a discrete set; recall that a countable union of sets of class $N_{B}$ is again of class $N_{B}([11$, p. 371$])$.

COROLlary 1. Let $D$ be a plane domain and let $E \subset D$ be a compact set of class $N_{B}$. Let $W$ be a Riemann surface which does not belong to $O_{A B}$ and whose ideal boundary contains no AB-removable point. Let $f: D \backslash E \rightarrow W$ be a holomorphic mapping. Then $f$ extends to a holomorphic mapping $f^{*}: D \rightarrow W$.

We now indicate how a recent result of Shiga [12, Theorem 2] can be obtained from Theorem 1. Following [12] we say that a Riemann surface $W$ is $C$-nondegenerate provided there exists $\varepsilon>0$ such that the Carathéodory length of every nontrivial smooth closed curve on $W$ exceeds $\varepsilon$.

Corollary 2 (Shiga [12]). Let $D$ be a plane domain and let $E \subset D$ be a compact set of class $N_{B}$. Let $W$ be a C-nondegenerate Riemann surface and $\widetilde{W}$ a Riemann surface which is a (possibly branched) covering surface over $W$ with the projection $\pi: \hat{W} \rightarrow W$. Suppose that for each $p$ in $W$ there exists a neighborhood 
$V$ of $p$ such that every component of $\pi^{-1}(V)$ is also C-nondegenerate. Let $f$ : $D \backslash E \rightarrow \widetilde{W}$ be a holomorphic mapping. Then $f$ extends to a holomorphic mapping $f^{*}: D \rightarrow \widetilde{W}$.

Proof. Since $W$ is $C$-nondegenerate, $W \notin \mathcal{O}_{A B}$. Of course, $\widetilde{W} \notin \mathcal{O}_{A B}$ too. Hence by Corollary 1 , it is enough to show that the ideal boundary of $\widetilde{W}$ does not possess $A B$-removable points. Assume it does have one, say $p$. Then there is a Riemann surface $\widetilde{W}^{\prime}$ such that $p \in \widetilde{W}^{\prime} \backslash \widetilde{W}$ and $\widetilde{W}^{\prime} \backslash \widetilde{W}$ is of class $N_{B}$ in $\widetilde{W}^{\prime}$. By Theorem 1, there exists a Riemann surface $W^{\prime} \supset W$ such that $\pi$ extends to a holomorphic mapping $\pi^{*}: \widetilde{W}^{\prime} \rightarrow W^{\prime}$.

Suppose first that $f^{*}(p) \in W$. Then, given any neighborhood $V$ of $f^{*}(p)$, we can find a parametric disc $(U, \varphi)$ of $\widetilde{W}^{\prime}$ such that $p \in U, U \cap \widetilde{W}$ is contained in a component of $\pi^{-1}(V)$ and $\partial U \cap\left(\widetilde{W}^{\prime} \backslash \widetilde{W}\right)=\varnothing$. Recalling that $U \cap\left(\widetilde{W}^{\prime} \backslash \widetilde{W}\right)$ is of class $N_{B}$ in $\widetilde{W}^{\prime}$ and making use of the relation $N_{B} \subset N_{D}$ as in [12] we see that $U \cap \widetilde{W}$ is not $C$-nondegenerate. Hence the same is true of the component of $\pi^{-1}(V)$ containing $U \cap \widetilde{W}$, contradicting the assumption.

There remains the case $f^{*}(p) \in W^{\prime} \backslash W$. Because $W^{\prime} \backslash W(\neq \varnothing)$ is of class $N_{B}$ in $W^{\prime}$, the argument given above shows that $W$ cannot be $C$-nondegenerate. This contradiction completes the proof.

THEOREM 2. Let $D$ be a plane domain and let $E \subset D$ be a compact set of class $N_{D}$. Let $W$ be a Riemann surface which does not belong to $\mathcal{O}_{M D^{*}}$, and let $f: D \backslash E \rightarrow W$ be a holomorphic mapping of bounded valence. Then there exists a Riemann surface $W^{\prime} \supset W$ such that

(a) $W^{\prime} \backslash W$ is of class $N_{D}$ in $W^{\prime}$ and

(b) $f$ extends to a holomorphic mapping $f^{*}: D \rightarrow W^{\prime}$.

Proof. Let $g \in M D *(W)$ be nonconstant. Since $f$ has bounded valence, $g \circ f \in M D^{*}(D \backslash E)$. By [3, Theorem 2], $g \circ f$ extends to a meromorphic function in $D$. Hence $C l(g \circ f ; z)$ is a singleton for each $z \in E$. It follows, as in the proof of Theorem 1 , that $f$ extends to a continuous mapping $f^{*}: D \rightarrow W^{*}$. Let $E^{\prime}$ stand for $\left(f^{*}\right)^{-1}(\beta)$. Then $f^{*}\left(E \backslash E^{\prime}\right) \subset W$. Since $f^{*} \mid D \backslash E^{\prime}$ is continuous and of bounded valence, $f^{*}$ is actually holomorphic in $D \backslash E^{\prime}$ [3, Theorem 2]. From now on the proof proceeds in complete analogy with the proof of Theorem 1. Hence we may omit the details.

Corollary. Let $D$ be a plane domain and let $E \subset D$ be a compact set of class $N_{D}$. Let $W$ be a Riemann surface which does not belong to $\mathcal{O}_{M D^{*}}$ and whose ideal boundary contains no AD-removable point. Let $f: D \backslash E \rightarrow W$ be a holomorphic mapping of bounded valence. Then $f$ extends to a holomorphic mapping $f^{*}$ : $D \rightarrow W$.

We conclude this note with two open problems.

(1) Let $D$ be a plane domain and let $E \subset D$ be a compact set of class $N_{B}$. Let $f$ be a holomorphic mapping of $D \backslash E$ into a Riemann surface $W$ and suppose 
that for some $z \in E C l(f ; z)$ is neither empty nor a singleton. Must then $\operatorname{Cl}(f ; z)$ coincide with $W$ ? More generally, one may ask whether such mappings have the localizable Iversen property [11, p. 365]. Note that the latter question seems to be open even in the case that $W$ is the Riemann sphere, while the former is of course trivial in this special case. The problem has relevance to Theorem 1 , because there are Riemann surfaces in $\mathcal{O}_{A B}$ with big or even arbitrary "holes" (Myrberg, Kuramochi).

(2) Is Theorem 2 true without the assumption that $W \notin \mathcal{O}_{M D^{*}}$ ?

\section{REFERENCES}

[1] L.V. Ahlfors And A. Beurling, Conformal invariants and function-theoretic null-sets, Acta Math., 83 (1950), 101-129.

[2] M. Heins, On Fuchsoid groups that contain parabolic transformations, Contributions to Function Theory, Tata Institute, Bombay (1960), 203-210.

[3] P. JäRvi, On the continuation of meromorphic functions, Ann. Acad. Sci. Fenn. Ser. A I Math., 12 (1987), 177-184.

[4] P. JARVI, Generalizations of Picard's theorem for Riemann surfaces, to appear.

[5] A. MARDEn, I. Richards AND B. Rodin, Analytic self-mappings of Riemann surfaces, J. Analyse Math., 18 (1967), 197-225.

[6] T. Nishino, Prolongements analytiques au sens de Riemann, Bull. Sci. Math. France, 107 (1979), 97-112.

[7] M. OHTsuka, On the behavior of an analytic function about an isolated boundary point, Nagoya Math, J., 4 (1952), 103-108.

[8] M. OHtsuka, Boundary components of abstract Riemann surfaces, Lectures on Functions of a Complex Variable (W. Kaplan, ed.), Ann Arbor (1955), 303-307.

[9] H. RengGli, Remarks on the Picard theorem, Lecture Notes in Math., vol. 1351, Springer-Verlag, Berlin (1988), 279-284.

[10] H.L. Royden, The Picard theorem for Riemann surfaces, Proc. Amer. Math. Soc., 90 (1984), 571-574.

[11] L. SaRio AND M. NAKAI, Classification theory of Riemann surfaces, SpringerVerlag, Berlin-Heidelberg-New York, 1970.

[12] H. SHIGA, On the boundary behavior of holomorphic mappings of plane domains to Riemann surfaces, J. Math. Kyoto Univ., to appear.

[13] M. Suzuki, Comportement des applications holomorphes autour d'un ensemble polaire, C. R. Acad. Sci. Paris, 304 (1987), 191-194.

Department of Mathematics

UNIVERSITY OF HELSINKI, SF-00100 HELSINKI 10, FinLAND 